\title{
Trastorno bipolar infantil: a propósito de un caso
}

\section{Pediatric bipolar disorder: on one case}

\author{
L. Pérez Fernández ${ }^{1,}$ A. Leache ${ }^{2}$, L. Ojer ${ }^{2}$
}

\section{RESUMEN}

Presentamos el caso de un varón de 14 años sin antecedentes de tratamiento psiquiátrico, aunque hace un año, tras una intervención quirúrgica de rodilla, presenta una época de mayor desánimo y retraimiento social. Desde hace tres meses se muestra más eufórico e hiperactivo, con conflictos con su familia y amigos.

Acude al Servicio de Urgencias de este hospital acompañado de su padre. Se solicita entonces valoración por parte del Servicio de Psiquiatría ante la presencia de síntomas maniacos (inquietud psicomotriz, verborrea, disforia y delirios de grandeza). Tras la valoración se decide ingreso en Unidad de Hospitalización Psiquiátrica para evaluación diagnóstica y tratamiento.

Durante el ingreso se diagnosticó al paciente de trastorno bipolar (en fase maniaca) y se instauró un tratamiento a largo plazo con un estabilizador del ánimo (ácido valproico) y un antipsicótico (risperidona). Tras el ingreso fue remitido a su Centro de Salud Mental para control del tratamiento y de la evolución de la enfermedad.

Palabras clave. Enfermedad bipolar. Enfermedad bipolar infantil. Tratamiento enfermedad bipolar.

\begin{abstract}
We present the case of a 14 year old male without antecendents of psychiatric treatment, although one year ago, following a surgical intervention on his knee, he presented a period of greater despondency and social withdrawal. Since three months ago he has been more euphoric and hyperactive, with conflicts with his family and friends.

He came to the Accidents and Emergencies Service of this hospital accompanied by his father. Evaluation by the Psychiatry Service was requested facing the presence of maniac symptoms (psychomotor anxiety, verbal incontinence, dysphoria and delusions of grandeur). Following evaluation it was decided to send him to the Psychiatric Hospitalization Unit for diagnostic evaluation and treatment.

During his hospital stay the patient was diagnosed as having bipolar disorder (in its maniac phase) and a long term treatment was initiated with a mind stabilizer (Valproic acid) and antipsychotic (Risperidone). Following the stay he was sent to the Mental Health Centre for follow up and evaluation of the disease.
\end{abstract}

Key words. Bipolar disease. Pediatric bipolar disease. Bipolar disease treatment.
1. Unidad de Hospitalización Psiquiátrica. Hospital de Navarra. Pamplona.

2. Unidad de Hospitalización Pisquiátrica. Hospital Virgen del Camino. Pamplona.

Recepción: 9 de diciembre de 2009

Aceptación provisional: 4 de enero de 2010

Aceptación definitiva: 29 de enero de 2010

\section{Correspondencia}

Lola Pérez Fernández

Paseo Santxiki, $134,1^{\circ} \mathrm{B}$

31192 Mutilva Alta (Navarra)

E-mail: iitxal80@hotmail.com 


\section{INTRODUCCIÓN}

La enfermedad bipolar es un trastorno psiquiátrico frecuente, crónico y de marcado componente genético que, generalmente, comienza en la adolescencia o en la edad adulta temprana. Esta enfermedad es un trastorno del humor caracterizado por episodios recurrentes de manía y/o hipomanía y episodios de depresión ${ }^{1}$.

Detectar un trastorno bipolar en niños y adolescentes resulta difícil por compartir síntomas con muchos otros trastornos, entre los que destacan trastorno por déficit de atención con hiperactividad (TDAH), trastornos de conducta y otros cuadros disruptivos.

El 8\% de los adultos afectados por trastorno bipolar tuvieron las primeras manifestaciones de la enfermedad antes de los 14 años, razón por la que se hace necesario alertar sobre este trastorno en la infancia y adolescencia.

En relación al tratamiento, es importante partir de la premisa de que el principal factor de riesgo reside en no tratar.

\section{CASO CLÍNICO}

Varón de 14 años de edad, sin antecedentes de tratamiento psiquiátrico, aunque hace un año y coincidiendo con una intervención quirúrgica de rodilla refiere una época de mayor desánimo y retraimiento social, no habiendo recibido ningún tratamiento farmacológico que pudiese inducir estos síntomas. Tampoco cuenta con antecedentes familiares de psicopatología.

Desde hace 3 meses su familia le nota más eufórico, hiperactivo, con múltiples planes y gastos económicos excesivos, lo que no es habitual en él.

Desde hace 15 días la sintomatología se ha exacerbado con conflictos con sus amigos y familia, con quienes se muestra muy disfórico. Refiere además que se va a hacer millonario, que aprende todo rápidamente y que posee una superioridad de conocimientos.

Acude a urgencias con la idea de hacerse una analítica para demostrar a sus padres que no consume tóxicos (la analítica de tóxicos en orina resulta negativa y no constan antecedentes de consumo de ninguna sustancia de abuso). El paciente se muestra inquieto y verborreico, con continuas bromas y risas. Comenta que se encuentra mejor que nunca. A los pocos minutos comienza a llorar.

En la exploración psicopatológica presenta hiperactividad, inquietud psicomotriz, ideación sobrevalorada de sí mismo y sus capacidades, ánimo exaltado con sensación subjetiva de bienestar, verborrea con presión al habla y discurso saltígrado, disforia con predominio en el ámbito familiar, desinhibición conductual y disminución de la necesidad de sueño.

\section{DISCUSIÓN}

En el DSM-IV (4 $4^{\mathrm{a}}$ edición del manual de diagnóstico de la Academia Americana de Psiquiatría) la enfermedad bipolar consta de cuatro subtipos:

1. Tipo I: con uno o más episodios de manía, o mixtos, generalmente acompañados de depresión mayor.

2. Tipo II: con uno o más episodios de depresión mayor y al menos un episodio de hipomanía.

3. Trastorno ciclotímico: caracterizado por al menos 2 años de numerosos periodos de hipomanía y de depresión que no cumplen criterios de manía y depresión mayor respectivamente.

4. Enfermedad bipolar sin especificar: con síntomas que no cumplen criterios completos de ningún subtipo anterior.

Muchos de los síntomas que corresponden a la manía son opuestos a los de la depresión (Tabla 1). Sin embargo, también comparten algunos de ellos como ira, irritabilidad, agitación e insomnio.

Mientras estos graves estados de ánimo se presentan durante periodos de semanas o meses en adultos, los niños experimentan cambios en su estado de ánimo en un mismo día. Las características más comunes consisten en bajo nivel de energía por las mañanas que siguen aumentando en el nivel de excitación hacia el final de la tarde o por la noche ${ }^{2}$. Son impulsivos y difíciles de controlar y no responden a ningún castigo $^{1}$. 
Tabla 1. Síntomas.

\begin{tabular}{ll}
\hline \multicolumn{1}{c}{ Síntomas de depresión } & \multicolumn{1}{c}{ Síntomas de manía } \\
\hline - Tristeza, llantos frecuentes o labilidad emocional & - Humor elevado, euforia desmedida e injustificada \\
- Estupor depresivo (inhibición motora muy & - Irritabilidad excesiva o cambios bruscos \\
marcada) & - Verborrea \\
- Ideas de suicidio o de muerte & - Insomnio \\
- Alteraciones del sueño (insomnio o excesiva & - Inquietud psicomotora o agitación \\
somnolencia) & - Delirio de grandeza y de omnipotencia \\
- Alteraciones del apetito & - Pensamiento superficial \\
- Irritabilidad & - Alucinaciones (en casos graves) \\
- Decaimiento psicofísico generalizado & - Pérdida o incremento del apetito \\
- Malos resultados escolares o cambios en la relación & - Desinhibición sexual \\
social & \\
\hline
\end{tabular}

Es necesario tener en cuenta que existen ciertos riesgos asociados al trastorno en niños y adolescentes, como son el fracaso escolar, el abuso de sustancias (principalmente en la pubertad) y el suicidio ${ }^{3}$.

La duración de los episodios (manía o depresión) varía entre individuos y no está relacionada con la gravedad, ya que hay episodios muy breves pero muy graves $y$ otros también breves pero muy leves. La respuesta al tratamiento también es una variable independiente de la gravedad de la enfermedad, variando asimismo de unos individuos a otros.

La enfermedad bipolar es igual de frecuente entre ambos sexos, con una prevalencia entre un 1 y un $2 \%$ en la población general. Sin embargo, la evolución del trastorno es diferente, siendo más predominantes los episodios de manía en varones y los de depresión en mujeres. Así mismo las mujeres tienen más probabilidad de virar de la depresión a la manía cuando reciben tratamiento con antidepresivos.

Según un estudio realizado por la Universidad de Navarra, durante un periodo de seis años, la edad media en el diagnóstico de enfermedad bipolar en niños y adolescentes es de 13,9 años, con un retraso en el diagnóstico de aproximadamente un año y medio. Las cifras extraídas de este estudio se asemejaban a los realizados en EEUU $^{4}$.

Es necesario resaltar la importancia de un diagnóstico correcto y precoz que posibilite la prescripción de un tratamiento, que va a minimizar las graves consecuencias de la no intervención. Así, se hace necesario considerar estos posibles casos de manía en las salas de urgencias, para realizar el diagnóstico diferencial en niños que presenten agitación psicomotriz.

El tratamiento consiste en estabilizantes del humor como el litio, el ácido valproico y la carbamacepina. También se usan los antipsicóticos con efecto estabilizador como la olanzapina, la quetiapina y la risperidona, siendo posible combinar ambos tipos de medicamentos.

Hay que prestar una especial atención a los niños con trastorno bipolar tratados con antidepresivos, porque existe un mayor riesgo de viraje (de la depresión a la manía), pudiendose volver mucho más irritables y con cambios de humor mucho más bruscos ${ }^{4}$.

Se torna de especial importancia en niños y adolescentes la estrecha supervisión de la toma del tratamiento ya que además la edad temprana en el inicio de la enfermedad supone uno de los factores de riesgo para el incumplimiento farmacológico.

Tras el diagnóstico será imprescindible el seguimiento ambulatorio de estos pacientes así como la hospitalización en casos en que se requiera un ajuste farmacológico ante la presencia de un episodio grave de descompensación.

Así, el caso que nos ocupa fue remitido tras el alta a su Centro de Salud Mental de referencia, donde se supervisará el tratamiento y la evolución de la enfermedad. 


\section{BIBLIOGRAFÍA}

1. Soutullo Esperón C. Depresión y enfermedad bipolar en niños y adolescentes. Eunsa 2005; 89-117.

2. NAMI. The nation's voice on mental illness. Trastorno bipolar en la infancia. Julio 2005. Disponible en www.nami.org (acceso 5 de enero de 2009).

3. Díaz Atienza J, Blánquez Rodríguez P. Trastorno bipolar en la infancia y adolescencia. Guía para padres de la Unidad de Salud Mental Infanto-Juvenil de Almería (acceso 5 de enero de 2009). Disponible en: http://www.cat-barcelona.com/pdf/biblioteca/trastorno-bipolar/1_-_trastorno_bipolar_en_la_infancia_y_ adolescencia.pdf

4. Soutullo CA, Escamilla-Canales I, Wozniak J, Gamazo-Garrán P, Figueroa-Quintana A, Biederman J. Pediatric bipolar disorder in a spanish sample: features before and at the time of diagnosis. J Affective Disorders 2009; 118: 39-47. 